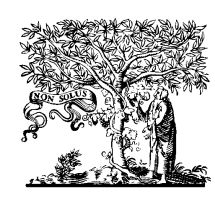

ELSEVIER

\title{
Effects of the dopamine D2 agonist, quinpirole, on time and number processing in rats
}

\author{
Angelo Santi*, Romina Coppa, Lori Ross \\ Department of Psychology, Wilfrid Laurier University, Waterloo, Ontario, Canada N2L 3C5
}

Received 10 March 2000; received in revised form 29 August 2000; accepted 19 September 2000

\begin{abstract}
In Experiment 1, rats were trained to discriminate discrete sound sequences that varied in time or number. On time trials, the number of sounds was held constant at 4 and the duration of the sound sequence was either 2 or $8 \mathrm{~s}$. On number trials, the duration of the sound sequence was held constant at $4 \mathrm{~s}$, and the number of sounds was either 2 or 8 . Psychophysical functions for time and number were obtained by presenting unreinforced sequences of intermediate duration or number. In agreement with previous research, sensitivity to variation in time was greater than variation in number. Rats received injections of the specific D2 agonist, quinpirole $(0.08 \mathrm{mg} / \mathrm{kg})$, or saline. Quinpirole significantly attenuated control by both time and number, but it did not increase behavioral estimates of time or number. In Experiment 2 , rats were given different dosages of quinpirole $(0.02,0.04$ or $0.06 \mathrm{mg} / \mathrm{kg})$. The steepness of the psychophysical functions for both time and number was reduced in a dose-related fashion without any evidence of an increase in the estimation of time or number. The similarity of the effect of quinpirole on both time and number processing is consistent with the idea that the same internal mechanism is used for timing and counting. However, it is not consistent with the idea that D2 dopamine agonists selectively increase the rate of the internal clock, which is assumed to underlie performance in a temporal bisection procedure. Quinpirole (at doses between $0.02 \mathrm{and} 0.08 \mathrm{mg} / \mathrm{kg}$ ) reduces sensitivity to time and number in a bisection procedure without altering the speed of the internal clock. (C) 2001 Elsevier Science Inc. All rights reserved.
\end{abstract}

Keywords: Quinpirole; D2 agonist; Dopamine; Time and number processes; Discrimination; Rats; Internal clock

Previous research has provided some support for the involvement of dopamine in the modification of the speed of an internal clock (Maricq et al., 1981; Meck, 1983, 1986, 1996; Meck and Church, 1983). The internal clock model (Meck, 1983, 1996; Meck and Church, 1983) depicts how an animal perceives and remembers the duration of an event and decides if it is comparable to previously reinforced durations. The model is composed of two stages: the clock stage, consisting of the pacemaker, switch, and accumulator; and the memory stage, which includes both reference and working memory. Closure of the switch allows pulses from the pacemaker to travel to an accumulator. The accumulated pulses on each trial are compared with previously reinforced durations stored in reference memory and a response decision is made. Increasing experimental evidence suggests that counting

\footnotetext{
* Corresponding author.

E-mail address: asanti@wlu.ca (A. Santi).
}

employs a similar internal mechanism, with the switch acting in an "event" mode rather than in the "run" and "stop" modes, necessary for timing (Breukelaar and Dalrymple-Alford, 1998, 1999; Meck and Church, 1983; Roberts and Boisvert, 1998; Roberts and Mitchell, 1994). Clock speed is thought to be regulated by dopaminergic activity in the basal ganglia, while cholinergic activity in the frontal cortex has been implicated in the memory stage (Meck, 1996). Evidence for an increase in clock speed is derived from the immediate leftward horizontal shift of the psychophysical function for time, which occurs after administration of a dopamine agonist (Meck, 1996). By relating the binding affinity of dopamine antagonists to their potency in producing a shift in psychophysical functions for time, it has been determined that D2 (nonadenylate cyclase-linked) dopamine receptor subtypes play a major role in controlling the clock component of the internal clock model (Meck, 1986).

However, there are other studies in both rats and pigeons that have failed to produce results consistent with this 
hypothesis (Lejeune et al., 1995; Rapp and Robbins, 1976; Santi et al., 1995; Stubbs and Thomas, 1974). Neither procedural details nor methods of analysis provide a basis for distinguishing between those studies that show dopaminergic effects on clock rate and those that do not. For example, drug dosage and type of drug can be ruled out because contradictory results have been obtained in studies in which these parameters were identical (Meck, 1983; Santi et al., 1995). In addition, previous exposure to the dopaminergic drug does not seem to be an important variable for two reasons. First, selective clock effects with no attenuation of temporal control have been obtained in rats that had prior exposure to methamphetamine (Meck, 1983), as well as those without prior exposure (Meck and Church, 1983). Secondly, prior exposure to the specific dopamine D2 agonist, quinpirole, has been manipulated and no major differences in quinpirole's effect on psychophysical time functions were observed between preexposed and nonpreexposed conditions (Stanford and Santi, 1998).

Two main types of behavioral procedures have been used in the study of drug effects and timing in animals: the temporal bisection procedure and the peak interval procedure. In the temporal bisection procedure, rats are trained to choose between the left and right levers following signals of different durations. In the peak interval procedure, rats are trained on a fixed interval schedule to respond for food after a specified signaled interval has elapsed. Quinpirole has produced a significant leftward shift of the response distributions of rats when a peak procedure is used (Frederick and Allen, 1996). However, when a temporal bisection procedure is used, the effects of quinpirole were found to be limited to a general disruption of attention to temporal signals (Stanford and Santi, 1998). The dose of quinpirole used in these studies differed. In the study using the peak procedure, the dose was $0.01 \mathrm{mg} / \mathrm{kg}$; whereas in the study using the temporal bisection procedure, the dose was 0.08 $\mathrm{mg} / \mathrm{kg}$. This suggests that quinpirole might have selective clock effects at low doses, but not at high doses.

The purpose of the present research was to clarify the effects of the specific dopamine D2 agonist, quinpirole, on temporal and numerical processing. In Experiment 1, rats were trained to discriminate discrete sound sequences that varied in time or number using a bisection procedure. Once the task was acquired, rats underwent five sessions of psychophysical testing under saline, followed by 15 sessions of testing under quinpirole $(0.08 \mathrm{mg} / \mathrm{kg})$, and, finally, five additional sessions of saline testing. In Experiment 2, rats were trained on the same time and number discrimination task, but underwent five sessions of psychophysical testing under saline, followed by testing with quinpirole at doses of $0.02,0.04$, or $0.06 \mathrm{mg} / \mathrm{kg}$. The psychophysical functions generated were then analyzed, firstly to compare the effects of quinpirole on the processing of time and number, and secondly, to determine whether quinpirole selectively increases the speed of the internal clock at any of the doses studied.

\section{Experiment 1}

Rats were trained to discriminate discrete sound sequences that varied in time or number. On time trials, the number of sounds was held constant at 4 , and the duration of the sound sequences was either 2 or $8 \mathrm{~s}$. On number trials, the duration of the sound sequence was held constant at $4 \mathrm{~s}$, and the number of sounds was 2 or 8 . After criterion was reached, the animals underwent psychophysical testing to measure the proportion of long responses made for time intervals of 2,3 , $4,5,6$, and $8 \mathrm{~s}$, and the proportion of many responses for 2-, 3-, 4-, 5-, 6-, and 8-signal presentations. Initially, five sessions of psychophysical testing with saline injections were given, followed by 15 sessions of psychophysical testing under the specific D2 agonist, quinpirole. Finally, five additional sessions of psychophysical testing under saline were given. The objectives of this study were: (1) to replicate earlier work indicating a general disruption of attention to temporal signals with a $0.08 \mathrm{mg} / \mathrm{kg}$ dose of quinpirole; (2) to determine if numerical processing was affected by quinpirole in the same way as temporal processing; and (3) to determine if the effect of quinpirole disappeared with continued testing under the drug.

\section{Materials and methods}

\subsection{Subjects}

Eight experimentally naive, male Long-Evans rats (Charles River, Canada), approximately 225-267 g in weight and 58-67 days of age when the experiment began, were individually housed in clear Plexiglas shoebox cages with continuous access to water. Fluorescent lights were illuminated on a 12-h light:12-h dark cycle (7 AM-7 PM). Experimental sessions were conducted during the light phase. During testing, they were fooddeprived and maintained at approximately $85-90 \%$ of their normal body weight with supplemental feeding of Purina Rat Chow shortly after the experimental session each day.

\subsection{Apparatus}

Four Coulbourn modular operant chambers (Model \#E10-10), individually housed in isolation chambers (Model \#E10-20) and equipped with baffled exhaust fans, were used. On the front wall of the chamber, two retractable levers (Model \#E23-07 in two of the boxes and Model \#E23-17 in the other two) were positioned on either side of a pellet feeder (Model \#E14-06) approximately $3 \mathrm{~cm}$ from the grid floor and $14 \mathrm{~cm}$ apart. The pellet feeder was placed in the center of the front wall with the opening approximately $3 \mathrm{~cm}$ from the floor of 
the chamber and provided access to $45 \mathrm{mg}$ pellets (Bioserve Universal Research Test Diets grain-based rodent pellets). A $2.9-\mathrm{kHz}$ tone module sonalert (Model \#E12-02) was mounted directly above each retractable lever. Onset of both sonalerts was the carrier of the temporal and numerical signals. A house light (Model \#E11-01, bulb \#SL1819x) positioned $6.5 \mathrm{~cm}$ directly above the pellet feeder and reflecting toward the ceiling of the chamber remained on throughout all sessions. All experimental events and response measures were controlled by a microcomputer located in the same room.

\subsection{Procedure}

\subsubsection{Pretraining}

Each rat received 10-15 sessions of combined magazine and lever training. The rats were placed in the chamber with both the left and right levers retracted. Each trial commenced with the entry of the left or right lever into the chamber. The lever remained extended until it was pressed or $60 \mathrm{~s}$ had elapsed, whichever occurred first. Either event resulted in delivery of a food pellet and retraction of the lever. Pellet delivery produced an audible "click" and the light in the magazine was illuminated for $0.5 \mathrm{~s}$. The houselight illuminated the chamber throughout each session. Each session consisted of 160 60-s trials. Once lever pressing was established, discrimination training began.

\subsubsection{Training}

Sound signals were produced by the simultaneous onset of both sonalerts. On time trials, the number of sounds was held constant at 4 and the duration of the sound sequence was either 2 or $8 \mathrm{~s}(4 \mathrm{f} / 2 \mathrm{~s}$ or $4 \mathrm{f} / 8 \mathrm{~s})$. On number trials, the total duration of the sound sequence was $4 \mathrm{~s}$, and the number of sounds was either 2 or 8 (2 f/ $4 \mathrm{~s}$ or $8 \mathrm{f} / 4 \mathrm{~s})$. The tone on-off duration was $0.25 \mathrm{~s}$ for the $4 \mathrm{f} / 2 \mathrm{~s}$ and $8 \mathrm{f} / 4 \mathrm{~s}$ sequence, and it was $1 \mathrm{~s}$ for the $4 \mathrm{f} /$ $8 \mathrm{~s}$ and $2 \mathrm{f} / 4 \mathrm{~s}$ sequences. Half of the rats were trained to press the left lever following $4 \mathrm{f} / 2 \mathrm{~s}$ or $2 \mathrm{f} / 4 \mathrm{~s}$ (i.e., classifying the signal as "short" or "few") and to press the right lever following $4 \mathrm{f} / 8 \mathrm{~s}$ or $8 \mathrm{f} / 4 \mathrm{~s}$ (i.e., classify the signal as "long" or "many"). The remaining rats were trained with the opposite contingencies. A correct response was reinforced with a food pellet. An incorrect response initiated a correction procedure. The correction procedure consisted of a 5-s delay prior to a representation of the same sound sequence. A correct response during a correction trial resulted in delivery of a food pellet, presentation of an intertrial interval (ITI), and progression to the next trial. The ITI duration varied randomly from trial to trial $(5,10,15,20$, or $25 \mathrm{~s})$. Only the first response choice on each trial was included in the calculation of response accuracy. Each training session consisted of 160 trials (80 time trials and 80 number trials) with a maximum session duration of $120 \mathrm{~min}$. Training continued until accuracy was at least $75 \%$ correct on both number trials and time trials for all rats.

\subsubsection{Predrug saline testing}

Psychophysical testing was conducted for five daily sessions. Twenty minutes prior to each test session, all rats received an intraperitoneal injection of $0.9 \%$ physiological saline in a volume of $1 \mathrm{ml} / \mathrm{kg}$. Each of the four training signals (4 f/2 s, 4 f/8 s, 2 f/4 s, 8 f/4 s) was presented with a probability of 0.125 on each trial. On the remaining trials, one of eight intermediate signals $(3,4,5$, or 6 events/ duration) was presented with equal probability (.0625). On time trials, the total number of signals was 4 , and the duration of the sequence was $3,4,5$, or 6 s. On number trials, the total duration of the sequence was $4 \mathrm{~s}$, and the number of intermediate signals was $3,4,5$, or 6 . The rats were still rewarded for responding correctly to the four training signals, but responses to either lever following intermediate signals never resulted in food. Each test session consisted of 160 trials (80 time trials and 80 number trials) with a maximum session duration of $120 \mathrm{~min}$.

\subsubsection{Quinpirole testing}

Psychophysical testing continued to be conducted for 15 daily sessions, but $20 \mathrm{~min}$ prior to these test sessions, all rats received an intraperitoneal injection of quinpirole hydrochloride (Research Biochemicals International, Natick, MA) at a dose of $0.08 \mathrm{mg} / \mathrm{kg}$ in a $0.9 \%$ saline vehicle and a volume of $1 \mathrm{ml} / \mathrm{kg}$. The $0.08 \mathrm{mg} / \mathrm{kg}$ dosage of quinpirole was the same as in our earlier study (Stanford and Santi, 1998), which was in turn based on previous research using a spatial delayed nonmatching-to-position task (Bushnell and Levin, 1993). Except for the injection of quinpirole, all details of psychophysical testing were the same as previously described.

\subsubsection{Postdrug saline testing}

Following completion of the psychophysical testing with quinpirole, animals received an additional five sessions of psychophysical testing with saline injections $20 \mathrm{~min}$ prior to the experimental session. All details of testing were the same as described above.

\section{Results and discussion}

The data from the 15 sessions of psychophysical testing under quinpirole were organized into three blocks of five sessions each. An analysis of variance (ANOVA) with dimension (time, number), block, and signal value as within-subjects factors indicated that there was no significant main effect of block $[F<1]$, nor did the block factor enter into any significant interactions [all values of $F \leq 1$ ]. This indicates that the effect of quinpirole on time and number processing remained constant across test sessions. There was no evidence of a weakening of quinpirole's 
effectiveness across the three blocks of five test sessions. Consequently, the quinpirole data were averaged across all 15 test sessions.

The mean percentage of long responses as a function of signal duration averaged across test sessions is presented in the top panel of Fig. 1. The mean percentage of many responses as a function of signal frequency averaged across test sessions is presented in the bottom panel of Fig. 1. For both the time and number dimension, the percentage of long/many responses increased as a function of signal value. Sensitivity to signal values was greater for the time dimension than for the number dimension. As a result, the time functions show steeper slopes than the number functions. The psychophysical functions were flattest under quinpirole, indicating less sensitivity to both time and number. Postdrug saline functions were also flatter than predrug saline functions, but not as flat as the quinpirole functions themselves, indicating a partial recovery in performance following the termination of quinpirole administration. The quinpiroleinduced decrease in the percentage of long/many responses
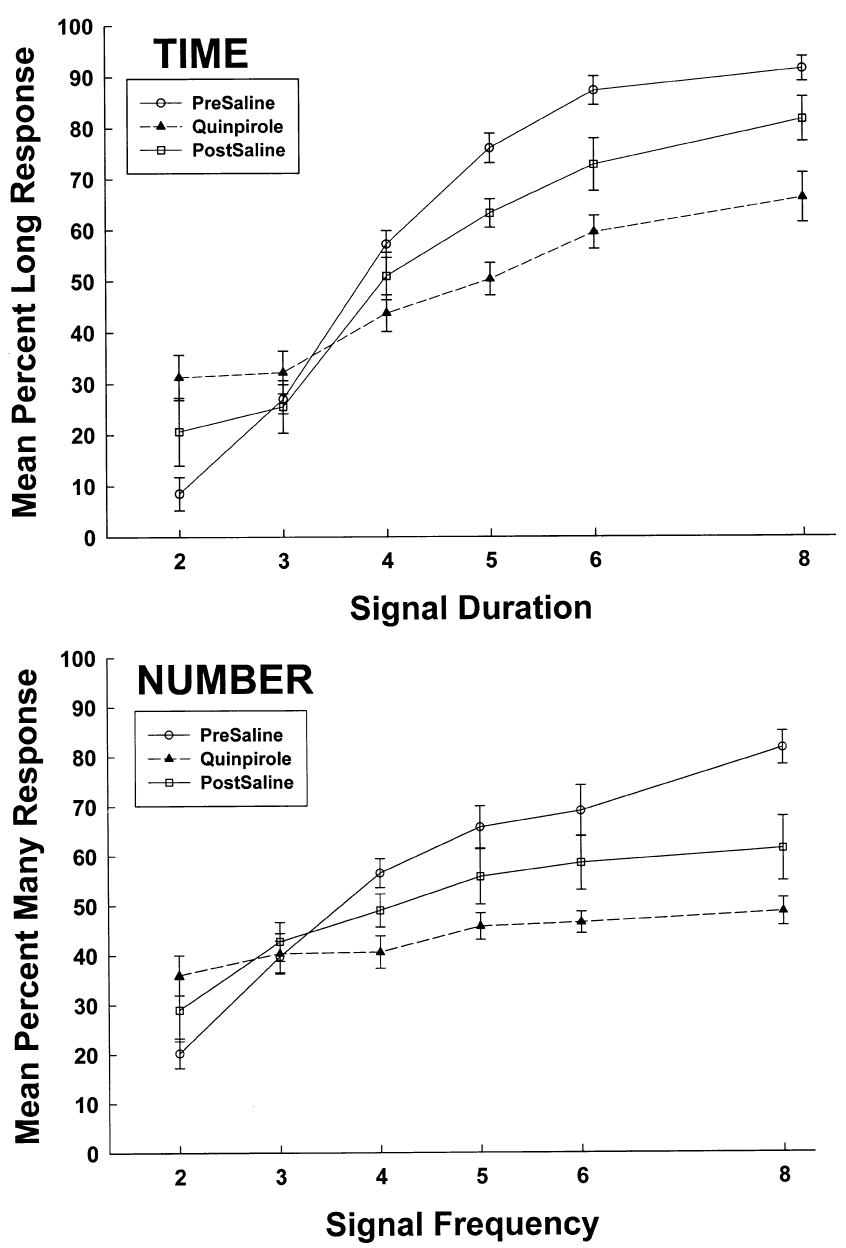

Fig. 1. Mean percentage of long/many responses as a function of signal value (time in the top panel, number in the bottom panel) averaged over five predrug saline test sessions, 15 quinpirole $(0.08 \mathrm{mg} / \mathrm{kg})$ test sessions, and five postdrug saline test sessions. Error bars represent S.E.M. was most severe at the upper signal values and least severe at signal value 3 .

An ANOVA revealed significant main effects of drug condition $[F(2,14)=9.38, P<.01]$ and of signal value $[F(5,35)=41.95, P<.01]$ as well as a significant Drug Condition $\times$ Signal interaction $[F(10,70)=19.30, P<.01]$. As is apparent in Fig. 1, the interaction resulted from a nonsignificant effect of drug condition at signal $3[F<1]$, while the effect of drug condition was significant at all other signal values $[F(2,14)=4.85$ to $48.20, P<.05]$. Neither the Drug Condition $\times$ Dimension nor the Drug Condition $\times$ Dimension $\times$ Signal interaction was significant $[F<2]$, indicating that quinpirole affected performance on both the time dimension and the number dimension equivalently.

There was also a significant main effect of dimension $[F(1,7)=6.01, P<.05]$, indicative of better performance on time trials than on number trials. The Dimension $\times$ Signal interaction was also significant $[F(5,35)=26.75, P<.01]$. Simple main effects analysis at each signal value revealed that this effect was due to a nonsignificant effect of dimension at signal value $4[F(1,7)=1.02]$, while the effect of dimension was significant at all other signal values $[F(1,7)=9.63$ to $43.41, P<.05]$. This indicates that the rats were more sensitive to variations in time than variations in number.

The point of subjective equality (PSE) was estimated from the psychophysical function for each rat by conducting linear regressions on the proportion of long and the proportion of many responses for each set of three adjacent points in the two functions (time and number). For each rat, the regression equation with the greatest slope for each dimension was used to estimate the PSE by calculating the signal duration or the signal frequency associated with $50 \%$ of the long or $50 \%$ of the many responses. These regression equations were also used to calculate the difference limen (DL), which represents the average difference between the signal duration or frequency associated with $75 \%$ long or many responses and the signal duration or frequency associated with $25 \%$ long or many responses. Finally, the Weber fraction (WF) was calculated as the DL/PSE. PSEs, DLs, and WFs were analyzed using repeated measures ANOVAs with dimension and drug condition as the within-subjects factors.

PSE analysis revealed no significant main effect of dimension $[F<1]$, since the mean PSE for time collapsed across drug conditions (4.14) was not significantly different from the mean PSE for number (4.46). When only the predrug saline PSEs were considered, single-sample $t$ tests revealed that the mean PSE for number (3.63) was significantly below the geometric mean of $4.0[t(7)=-3.56$, $P<.01]$, while the mean PSE for time (3.81) was not $[t<2]$. While the PSEs were higher under quinpirole (time: 4.91, number: 4.53) than under predrug saline, the effect of drug condition was not statistically significant $[F<1]$ because of high variability in the estimated PSEs under quinpirole. For three rats, quinpirole flattened the psycho- 
physical functions to such an extent that the estimated PSEs were outside of the range of values tested. When the data were reanalyzed only for the five rats with estimated PSEs between the training values of 2 and 8 , the effect of drug condition on the PSE was statistically significant $[F(2,8)=8.76, P<.01]$. There was no significant two-way interaction of Dimension $\times$ Drug Condition in either analysis $[F<1]$.

Analysis of the DLs revealed an effect of dimension that approached significance $[F(1,7)=5.18, P<.06]$, with discrimination of time being slightly better than discrimination of number. There was a significant main effect of drug condition $[F(2,14)=4.50, P<.05]$, indicative of poorer discrimination under quinpirole than under saline. The two-way interaction of Dimension $\times$ Drug Condition was not significant $[F=3.02]$.

Analysis of the WFs revealed no significant main effect of dimension $[F<1]$, indicating equivalent discrimination of time and number signals. The main effect of drug condition was also nonsignificant $[F<1]$. Finally, the twoway interaction between dimension and drug condition was nonsignificant $[F<1]$.

The flatter psychophysical functions for number than time demonstrate better control by temporal stimuli than by numerical stimuli. This is consistent with the literature for rats (Breukelaar and Dalrymple-Alford, 1998, 1999) and pigeons (Roberts and Boisvert, 1998), although it raises questions for the idea of a common processing mechanism for time and number (Meck and Church, 1983). Some researchers have hypothesized that the same internal mechanism could be involved, but superior temporal processing occurs as a result of temporal information being considered before numerical information at the comparator phase of the internal clock model (Breukelaar and Dalrymple-Alford, 1998). As a result, when both temporal and numerical information are available, the temporal information will control responding, and animals are less capable of responding on the basis of number. The lack of a significant interaction between drug condition and dimension in the analysis of the psychophysical functions, the DLs, and the WFs provides further support for the hypothesis that time and number processings are controlled by a similar internal mechanism (Meck, 1983) because time and number were affected equivalently by quinpirole.

The psychophysical functions shown in Fig. 1 suggest that quinpirole causes a performance deficit from which the rats recover gradually once injections of quinpirole are terminated, since postdrug saline functions were flatter relative to predrug saline functions. When predrug saline PSEs were considered, the mean PSE for time did not differ significantly from the geometric mean of 4.0 , while the mean PSE for number was significantly below it. Although PSEs for time are often reported to be at the geometric mean (Breukelaar and Dalrymple-Alford, 1999; Church and Deluty, 1977; Meck, 1983), the results are less consistent for number (Fetterman, 1993; Fetterman et al., 1986;
Martin-Iverson et al., 1988). The flatter psychophysical functions during drug testing suggest that quinpirole impaired performance on both time and number discriminations without selectively increasing the speed of the internal clock. This replicates the results of an earlier study on time discriminations in our laboratory (Stanford and Santi, 1998) and suggests that this selective dopamine agonist has similar effects on number discriminations, as would be expected if time and number are indeed processed using similar internal mechanisms.

\section{Experiment 2}

The lack of evidence for an increase in that speed of the internal clock in Experiment 1 could be attributed to the dose of quinpirole used. It is possible that a dose of $0.08 \mathrm{mg} /$ $\mathrm{kg}$ is high enough that any effect on clock speed is masked by the general disruption in performance that results. Experiment 2 was designed to test this hypothesis by looking at the effect of lower doses of quinpirole on time and number processing.

\section{Materials and methods}

\subsection{Subjects}

Twelve male Long-Evans rats served as subjects. As in Experiment 1, they were housed in individual stainless steel cages in a room maintained on a 12-h light:12-h dark cycle, had free access to water at all times, and were maintained at $85-90 \%$ of their ad libitum body weights. Of the 12 , four rats had previously been subjects in Experiment 1, four in a similar experiment involving methamphetamine $(1.5 \mathrm{mg} / \mathrm{kg})$ injections, and the remaining four in a similar experiment involving scopolamine $(0.1 \mathrm{mg} / \mathrm{kg})$ injections.

\subsection{Apparatus}

The apparatus were identical to those used in Experiment 1 .

\subsection{Procedure}

Rats were given 11 days of discrimination training as described in Experiment 1 to reestablish baseline performance (at least $75 \%$ correct on both dimensions for each rat). This was followed by three testing cycles each consisting of 5 days of psychophysical testing with saline (as described in Experiment 1), 5 days of psychophysical testing with quinpirole, and reestablishment of baseline performance. During drug testing sessions, 20 min prior to the experimental session, the animals received an intraperitoneal injection of either $0.02,0.04$, or $0.06 \mathrm{mg} / \mathrm{kg}$ quinpirole in a $0.9 \%$ saline vehicle (volume of $1 \mathrm{ml} / \mathrm{kg}$ ). For the 
first cycle of drug testing, one third of the animals received $0.02 \mathrm{mg} / \mathrm{kg}$ quinpirole (Group 1), one third received 0.04 $\mathrm{mg} / \mathrm{kg}$ quinpirole (Group 2), and the final third received $0.06 \mathrm{mg} / \mathrm{kg}$ quinpirole (Group 3). These groups were counterbalanced on the basis of previous drug experience. After the first cycle of drug testing, a 9-day baseline discrimination training was conducted before beginning Cycle 2. For the second cycle of drug testing, Group 1 received $0.04 \mathrm{mg} / \mathrm{kg}$ quinpirole, Group 2 received $0.06 \mathrm{mg} /$ $\mathrm{kg}$ quinpirole, and Group 3 received $0.02 \mathrm{mg} / \mathrm{kg}$ quinpirole. Three days of discrimination training were then given to reestablish baseline levels of performance. For the final cycle of drug testing, each group received the dose of quinpirole they had not received in the previous two cycles. Other than the changes noted here, all other aspects of baseline training and psychophysical testing were identical to Experiment 1.

\section{Results and discussion}

The mean percentage of long/many responses as a function of signal value averaged across test sessions is presented in Fig. 2 (time in the top panel, number in the bottom panel). For both time and number, the percentage of long/many responses increased as a function of signal value. As in Experiment 1, accuracy was better on time trials than on number trials and, as a result, the time functions show steeper slopes than the number functions. For both time and number, quinpirole disrupted accuracy on the task, as evidenced by the flatter psychophysical functions. The impairment was least severe for the $0.02 \mathrm{mg} / \mathrm{kg}$ dose, and essentially identical for the 0.04 and $0.06 \mathrm{mg} / \mathrm{kg}$ doses.

An ANOVA revealed significant main effects of dose $[F(3,33)=43.29, P<.01]$, of signal value $[F(5,55)=229.53$, $P<.01]$, as well as a significant dose by signal interaction $[F(15,165)=20.24, P<.01]$. As is apparent in Fig. 2, the interaction was the result of a decrease in the magnitude of the signal effect with increasing doses of quinpirole. That is, as dose increased, the functions became flatter $[F(5,55)=600.28,113.62,91.79$, and 58.23, $P<.01$, from the lowest $(0 \mathrm{mg} / \mathrm{kg})$ to the highest $(0.06 \mathrm{mg} / \mathrm{kg})$ dose of quinpirole, respectively]. In addition, the effect of dose was weakest at signal value 3 , although it was still statistically significant $[F(3,33)=3.28, P<.05]$. As in Experiment 1, neither the Dose $\times$ Dimension $[F<2]$ nor the Dose $\times$ Dimension $\times$ Signal interaction was significant $[F<1]$, indicating that regardless of dose, quinpirole affected time and the number processing equivalently.

There was a significant main effect of dimension $[F(1,11)=7.48, P<.05]$, indicative of better performance on the time dimension than on the number dimension. The Dimension $\times$ Signal interaction was also statistically significant $[F(5,55)=33.41, P<.01]$. Simple main effects analysis indicated that this was due to a nonsignificant effect of dimension at signal value $4[F<1]$, while the effect of
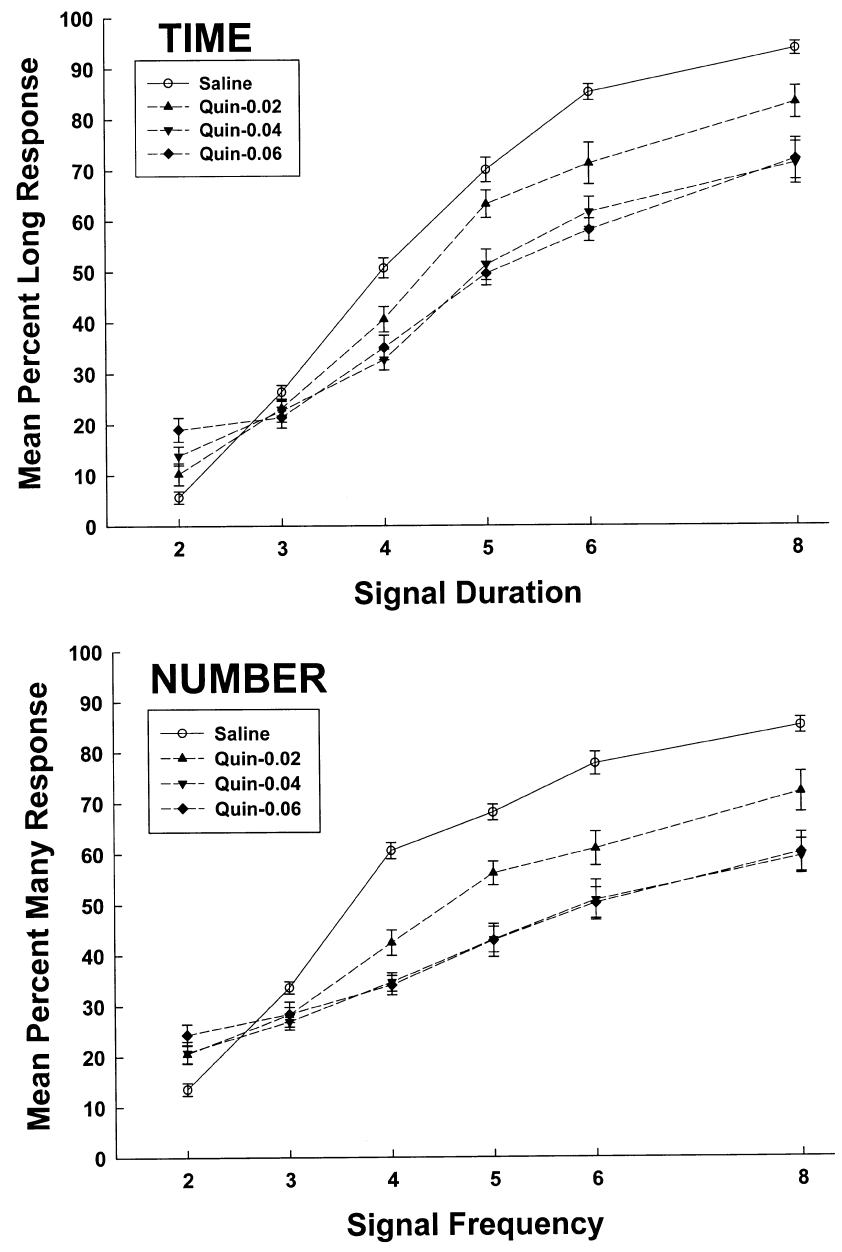

Fig. 2. Mean percentage of long/many responses as a function of signal value (time in the top panel, number in the bottom panel) for testing under saline, $0.02,0.04$, and $0.06 \mathrm{mg} / \mathrm{kg}$ quinpirole. Error bars represent S.E.M.

dimension was significant at all other signal values $[F(1,11)=14.38$ to $97.09, P<.01]$. These findings replicate those reported in Experiment 1.

PSEs, DLs, and WFs were calculated as described in Experiment 1 for the saline, 0.02, 0.04, and $0.06 \mathrm{mg} / \mathrm{kg}$ time and number functions for each rat. The PSEs, DLs, and WFs were analyzed using repeated measures ANOVAs with dose and dimension as the within-subjects factors.

PSE analysis revealed significant main effects of dose $[F(3,33)=32.47, P<.01]$, of dimension $[F(1,11)=11.47$, $P<.01]$, as well as a significant Dose $\times$ Dimension interaction $[F(3,33)=5.23, P<.01]$. A simple main effects analysis indicated that the effect of dose on the PSE was significant both for time $[F(3,33)=25.61, P<.01]$ and for number $[F(3,33)=23.33, P<.01]$. For both dimensions, as the dose of quinpirole increased, the mean PSE increased from 3.99 to 5.00 for time, and from 3.95 to 5.78 for number. While there was no difference in the PSE for time and number under saline, or with the $0.02 \mathrm{mg} / \mathrm{kg}$ dose of quinpirole $[F<2.65]$, at the two higher doses, the PSE for time was significantly less than the PSE for number $[F(1,11)=6.46$ and $12.59, P<.05]$. 
Analysis of the DLs revealed a significant main effect of dose $[F(3,33)=12.59, P<.01]$, indicating decreased discriminability as the dose of quinpirole increased. The main effect of dimension was also significant $[F(1,11)=17.89$, $P<.01]$, since the mean DL collapsed across the doses of quinpirole for number (2.11) was significantly higher than the mean DL for time (1.34). As in Experiment 1, this indicates that the rats exhibited greater sensitivity to time than to number. The Dose $\times$ Dimension interaction was not significant $[F(3,33)=2.24]$.

Analysis of the WFs followed the same patterns as the DL analysis. There was a main effect of dose $[F(3,33)=5.28$, $P<.05]$ as a result of poorer discrimination with higher doses of quinpirole. The main effect of dimension was also significant $[F(1,11)=29.30, P<.01]$ due to poorer discrimination on number trials than on time trials. The dose by dimension interaction was not significant $[F<1]$.

Eight of the rats used in this experiment had been used in a previous dopaminergic agonist study of the same design (four from Experiment 1 and four from a study involving a $1.5 \mathrm{mg} / \mathrm{kg}$ dose of methamphetamine). Because of concern regarding the possible effect of previous exposure to dopaminergic agonists, the psychophysical function data were reanalyzed with previous drug exposure as a factor. In this analysis, the grouping factor was based on whether the rats had previous exposure to dopaminergic agonist drugs (Group QuinpMeth, $N=8$ ) or scopolamine (Group Scop, $N=4$ ). Reanalysis of the psychophysical function data confirmed that previous drug exposure was not an important factor in the effect of quinpirole on the psychophysical functions. The main effect of group was not significant $[F<1]$, and the group factor did not enter into significant interactions with any of the other factors in the analysis [all values of $F \leq 1]$. The reanalysis again revealed significant main effects of dose $[F(3,30)=34.10, P<.01]$, of signal value $[F(5,50)=188.63, P<.01]$, as well as a significant dose by signal interaction $[F(15,150)=17.05, P<.01]$. There was also a significant main effect of dimension $[F(1,10)=7.05, P<.05]$ and a Dimension $\times$ Signal interaction $[F(5,50)=29.63, P<.01]$.

In this experiment, the three doses of quinpirole flattened the psychophysical functions for time and number compared to those obtained under saline. This occurred regardless of whether the rats had been previously exposed to either dopaminergic agonists or to scopolamine. This result is consistent with previous research showing that rats that had experienced quinpirole prior to testing showed the same flattening of psychophysical time functions as those rats that had no prior quinpirole experience (Stanford and Santi, 1998). It is also consistent with previous studies, which show that methamphetamine has the same effect on psychophysical time functions regardless of whether rats had prior exposure to methamphetamine (Meck, 1983), or no prior exposure (Meck and Church, 1983). Thus, prior exposure to dopaminergic agonists does not appear to be an important factor in determining how these drugs affect psychophysical time and number functions obtained in a bisection procedure.

Increased doses of quinpirole also resulted in an increase in the PSEs. The fact that quinpirole increased PSEs for time and number is inconsistent with the hypothesis that dopaminergic agonists increase clock speed (Meck, 1983, 1996) because an increase in clock speed should result in a decrease in the PSE. The increase in the PSEs would be more consistent with a slowing of the internal clock than an increase in clock speed. These data are consistent with previous temporal bisection studies, indicating that quinpirole (at doses between 0.02 and $0.08 \mathrm{mg} / \mathrm{kg}$ ) reduces the accuracy of temporal discriminations; however, it does not increase the speed of the internal clock (Stanford and Santi, 1998).

\section{General discussion}

Experiment 1 accomplished three objectives. It replicated earlier work (Stanford and Santi, 1998) indicating that attention to temporal signals is disrupted by a $0.08 \mathrm{mg} / \mathrm{kg}$ dose of quinpirole. It also demonstrated that numerical processing is affected by quinpirole in the same way as temporal processing. Finally, it demonstrated that the disruptive effects of quinpirole do not diminish with repeated exposure to the drug over 15 sessions of testing. Experiment 2 indicated that the effect of quinpirole on time and number processing is dose-dependent in a quantitative, rather than a qualitative way. That is, although the different doses of quinpirole disrupted processing to different degrees, the effects are limited to a general disruption of attention, and at neither the high nor the low doses is there any evidence of an increase in clock speed. Experiment 2 also indicated that the disruptive effects of quinpirole were the same regardless of whether rats had previous exposure to dopaminergic agonists or to scopolamine. This is consistent with previous research (Stanford and Santi, 1998) in showing that prior exposure to dopaminergic agonists does not appear to be an important factor in determining whether quinpirole produces a flattening, as opposed to a horizontal shift in psychophysical time functions.

In both experiments, the PSEs were significantly higher for both the time and the number dimension under quinpirole. This suggests that in addition to disrupting attention to time and number, quinpirole may actually slow down the internal clock. However, one would expect that clock effects would be attenuated as rats learn to rescale time and number under repeated exposure to the quinpirole. There was no evidence of attenuation over 15 sessions of testing in Experiment 1. Rescaling of time under dopaminergic drugs usually occurs within about seven sessions of training (see Meck, 1996), so it seems unlikely that 15 sessions of training under quinpirole were insufficient to detect a rescaling of either time or number. Thus, it appears that the increase noted in the PSEs for time and number may be 
a consequence of the loss of discriminative control produced by quinpirole rather than a clock effect.

In both experiments, analyses of the psychophysical functions, the DLs, and the WFs failed to reveal a significant Dose $\times$ Dimension interaction. This was also true of the PSE analysis in Experiment 1, but not in Experiment 2. Overall, it seems reasonable to conclude that time and number processings were affected equivalently by quinpirole. This is consistent with the hypothesis that time and number processing is controlled by a similar internal mechanism (Meck and Church, 1983).

In both experiments, the psychophysical functions indicated better control by temporal stimuli than by numerical stimuli. This replicates previous results for rats (Breukelaar and Dalrymple-Alford, 1998, 1999) and for pigeons (Roberts and Boisvert, 1998). Although the idea of a common processing mechanism for time and number (Meck and Church, 1983) may seem inconsistent with this finding, some researchers have hypothesized that the same internal mechanism could be involved if temporal information is evaluated before numerical information at the comparator phase of the internal clock model (Breukelaar and Dalrymple-Alford, 1998). As a result, when both temporal and numerical information are available, the temporal information will control responding and animals become less proficient at responding on the basis of number.

One potential limitation of the present research is the range of doses $(0.02-0.8 \mathrm{mg} / \mathrm{kg})$ that was studied. Previous research using a peak procedure (Frederick and Allen, 1996) reported that a $0.01 \mathrm{mg} / \mathrm{kg}$ dose of quinpirole significantly reduced peak time. While this finding appears consistent with dopamine agonists producing an increase in clock speed, it should be noted that this study (Frederick and Allen, 1996) failed to find a relationship between affinity for the $\mathrm{D} 2$ receptor and the decrease in peak time, and it also failed to detect a significant increase in peak time when D1 and D2 antagonists were administered. Nevertheless, some readers might wonder whether a $0.01 \mathrm{mg} / \mathrm{kg}$ dose of quinpirole would have produced a leftward shift of timing functions in our temporal bisection procedure. This assumes that the effects of a given dose of quinpirole would be the same in a free operant psychophysical task like the peak procedure and a retrospective timing task like temporal bisection. There is increasing evidence that this assumption is unwarranted. The effect of drugs on timing do not appear to be the same in free operant psychophysical tasks like the peak procedure and in temporal bisection tasks (Chiang et al., 2000). Although D-amphetamine often produces leftward shifts of psychophysical functions in free operant procedures (Chiang et al., 2000; Frederick and Allen, 1996; Kraemer et al., 1997; Maricq et al., 1981; Meck, 1996), similar doses fail to reduce the bisection point in temporal bisection tasks (Chiang et al., 2000; Stubbs and Thomas, 1974). Research involving the effects of central 5HT depletion has also shown that the same drug can have qualitatively different effects on timing behavior in different types of timing procedures (Al-Ruwaitea et al., 1997). The failure of D-amphetamine to reduce bisection points is consistent with a number of studies which have not found evidence for an involvement of dopaminergic mechanisms in the internal clock assumed to underlie performance in temporal bisection tasks (Chiang et al., 2000; Lejeune et al., 1995; Rapp and Robbins, 1976; Santi et al., 1995; Stanford and Santi, 1998; Stubbs and Thomas, 1974).

In summary, the accuracy of both temporal and numerical discriminations was reduced in a dose-related fashion by the selective dopamine D2 agonist, quinpirole. The similarity of the effect of quinpirole on both time and number processing is consistent with the idea that the same internal mechanism is used for timing and counting. However, it is not consistent with the idea that selective D2 dopamine agonists increase the rate of an internal clock, which is assumed to underlie performance in a temporal bisection procedure. The data are consistent with previous research indicating that quinpirole (at doses between 0.02 and $0.08 \mathrm{mg} / \mathrm{kg}$ ) attenuates temporal control in a bisection procedure without increasing the speed of the internal clock.

\section{Acknowledgments}

This research was supported by a grant from the Natural Sciences and Engineering Research Council of Canada (OGPOOD6378) to A. Santi, and by a Natural Sciences and Engineering Research Council of Canada Undergraduate Student Research Award to L. Ross. The data from Experiment 1 are based on a portion of a master's thesis submitted by R. Coppa to Wilfrid Laurier University. Romina Coppa is currently in the Department of Neuroscience at the University of Western Ontario, London, Ontario, and Lori Ross is currently at the Institute of Medical Sciences, University of Toronto, Toronto, Ontario. The authors thank Marion Corrick for her technical assistance.

\section{References}

Al-Ruwaitea ASA, Al-Zahrani SSA, Ho M-Y, Bradshaw CM, Szabadi E. Effect of central 5-hydroxytryptamine depletion on performance in the 'time-left' procedure: further evidence for a role of the 5-hydroxytryptaminergic pathways in behavioural 'switching'. Psychopharmacology 1997;134:179-86.

Breukelaar JWC, Dalrymple-Alford JC. Timing ability and numerical competence in rats. J Exp Psychol: Anim Behav Proc 1998;24:84-97.

Breukelaar JWC, Dalrymple-Alford JC. Effects of lesions to the cerebellar vermis and hemispheres on timing and counting in rats. Behav Neurosci 1999;113:78-90.

Bushnell PJ, Levin ED. Effects of dopaminergic drugs on working and reference memory in rats. Pharmacol, Biochem Behav 1993;45:765-76.

Chiang T-J, Al-Ruwaitea ASA, Mobini S, Ho M-Y, Bradshaw CM, Szabadi E. The effect of D-amphetamine on performance on two operant timing schedules. Psychopharmacology 2000;150:170-84.

Church RM, Deluty MZ. Bisection of temporal intervals. J Exp Psychol: Anim Behav Proc 1977;3:216-28. 
Fetterman JG. Numerosity discrimination: both time and number matter. $\mathrm{J}$ Exp Psychol: Anim Behav Proc 1993;19:149-64.

Fetterman JG, Dreyfus LR, Stubbs DA. Scaling of response-based events. J Exp Psychol: Anim Behav Proc 1986;11:388-404.

Frederick DL, Allen JD. Effects of selective dopamine D1 and D2 agonists and antagonists on timing performance in rats. Pharmacol, Biochem Behav 1996;53:759-64.

Kraemer PJ, Randall CK, Dose JM, Brown RW. Impact of D-amphetamine on temporal estimation in pigeons tested with a production procedure. Pharmacol, Biochem Behav 1997;58:165-73.

Lejeune H, Hermans I, Mocaer E, Rettori M, Poignant J, Richelle M. Amineptine, response timing and time discrimination in the albino rat. Pharmacol, Biochem Behav 1995;51:165-73.

Maricq AV, Roberts S, Church RM. Methamphetamine and time estimation. J Exp Psychol: Anim Behav Proc 1981;7:18-30.

Martin-Iverson MT, Fibiger HC, Wilkie DM. Alteration in the perception of food quantity by rats induced by manipulations of hunger and food sweetness. Learn Motiv 1988;19:44-65.

Meck WH. Selective adjustment of the speed of internal clock and memory storage processes. J Exp Psychol: Anim Behav Proc 1983;9:171-201.

Meck WH. Affinity for the dopamine D2 receptor predicts neuroleptic potency in decreasing the speed of an internal clock. Pharmacol, Biochem Behav 1986;25:1185-9.
Meck WH. Neuropharmacology of time perception. Brain Res Cognit Brain Res 1996;3:227-42.

Meck WH, Church RM. A mode control model of counting and timing processes. J Exp Psychol: Anim Behav Proc 1983;9:320-34.

Rapp DL, Robbins TW. The effects of D-amphetamine on temporal discrimination in the rat. Psychopharmacology 1976;51:91-100.

Roberts WA, Boisvert MJ. Using the peak procedure to measure timing and counting processes in pigeons. J Exp Psychol: Anim Behav Proc 1998;24:416-30.

Roberts WA, Mitchell S. Can a pigeon simultaneously process temporal and numerical information? J Exp Psychol: Anim Behav Proc 1994; 20:66-78.

Santi A, Weise L, Kuiper D. Amphetamine and memory for event duration in rats and pigeons: disruption of attention to temporal samples rather than changes in the speed of the internal clock. Psychobiology 1995;23: $224-32$.

Stanford L, Santi A. The dopamine D2 agonist, quinpirole, disrupts attention to temporal signals without selectively altering the speed of the internal clock. Psychobiology 1998;26:258-66.

Stubbs DA, Thomas JR. Discrimination of stimulus duration and D-amphetamine in pigeons: a psychophysical analysis. Psychopharmacologia $1974 ; 36: 313-22$ 